\title{
Extreme Male Color Polymorphism Supports the Introduction of Multiple Native-range Panther Chameleon (Furcifer pardalis) Lineages to Florida, USA
}

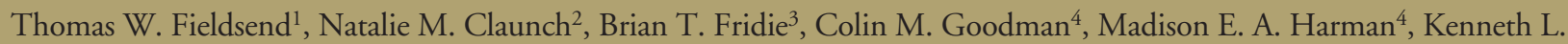
Krysko $^{5}$, Christopher J. Raxworthy ${ }^{6}$, Christina M. Romagosa ${ }^{4}$, and Timothy M. Collins ${ }^{1}$

\author{
${ }^{1}$ Department of Biological Sciences, Florida International University, Miami, Florida, USA (thomasfieldsend@hushmail.com) \\ ${ }^{2}$ School of Natural Resources and Environment, University of Florida, Gainesville, Florida, USA \\ ${ }^{3}$ Department of Biology, Eckerd College, Saint Petersburg, Florida, USA \\ ${ }^{4}$ Department of Wildlife Ecology and Conservation, University of Florida, Gainesville, Florida, USA \\ ${ }^{5}$ Division of Herpetology, Florida Museum of Natural History, Gainesville, Florida, USA \\ ${ }^{6}$ Division of Vertebrate Zoology, Department of Herpetology, American Museum of Natural History, New York, New York, USA
}

$\mathrm{T}_{\mathrm{s}}^{\mathrm{h}}$

he state of Florida, USA, is home to more non-native species of amphibians and reptiles than anywhere else on Earth (Krysko et al. 2016); many of these show unambiguous evidence of having derived from multiple geneti-

Background white, gray, or light red-brown, with red or brown bars?

No

Yes

Yellow posterior lip and green or green/red eyelids?
Yes

No
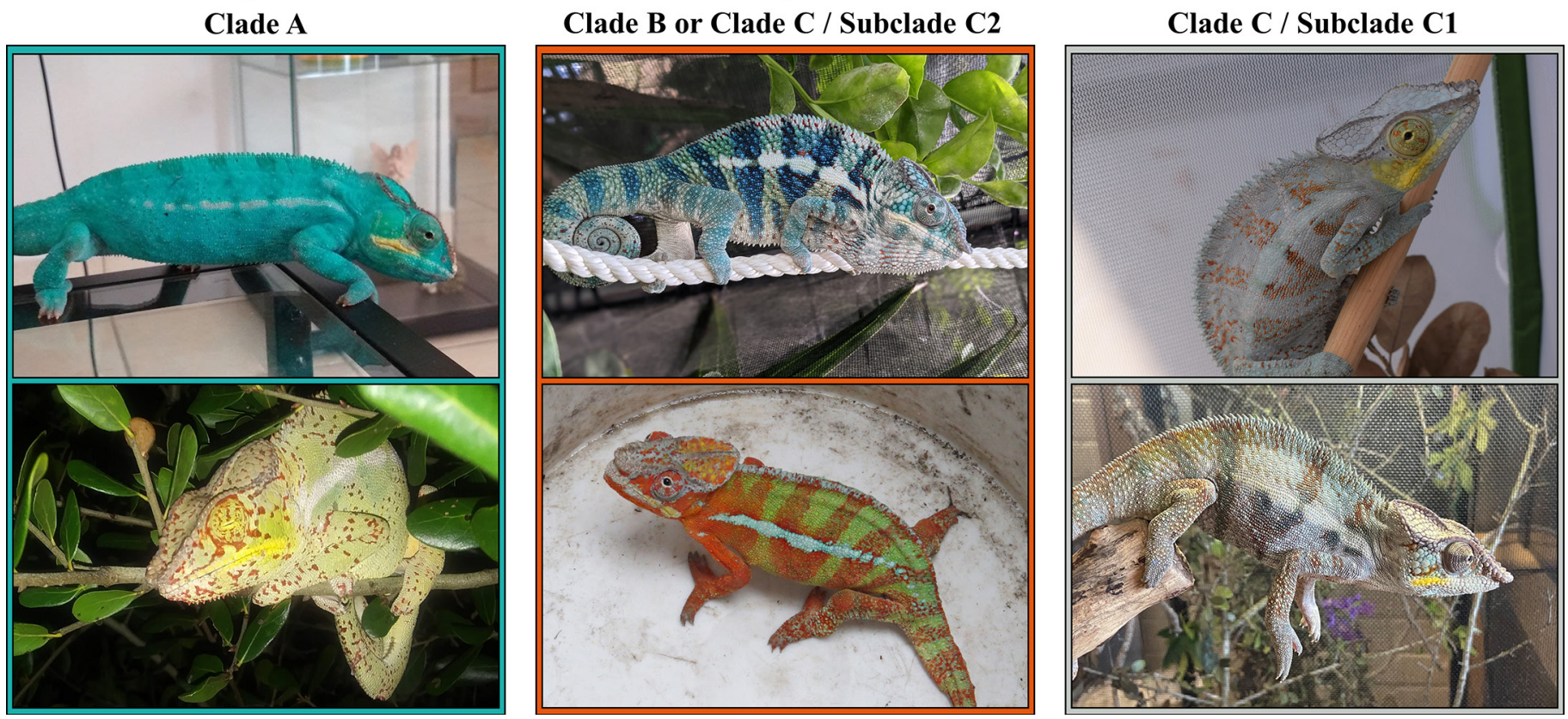

Fig. 1. Modified version of the identification key from Grbic et al. (2015) used in this study to determine the probable clade of origin of adult male Panther Chameleons (Furcifer pardalis) from Florida, USA $(\mathrm{n}=20)$. Note that the key does not allow for definitive assignment to Clade B or Clade C/Subclade C2. Representative images: Clade A (upper panel), Homestead, Miami-Dade County, photograph by Thomas W. Fieldsend; Clade A (lower panel), Babson Park, Polk County, UF-Herpetology image voucher 190325a; Clade B or Clade C/Subclade 2 (upper panel), Orange County, photograph by Natalie M. Claunch; Clade B or Clade C/Subclade 2 (lower panel), Coconut Creek, Broward County, UF-Herpetology image voucher 170801a; Clade C/Subclade C1 (upper panel), Sarasota County, photograph by Colin M. Goodman; Clade C/Subclade C1 (lower panel), Orange County, photograph by Brian T. Fridie. 
cally distinct native-range lineages (e.g., Kolbe et al. 2007; Fieldsend et al. 2021). When distinct lineages occur in sympatry, hybridization or admixture can result, sometimes leading to elevated population-level genetic diversity (Kolbe et al. 2007; Dlugosch and Parker 2008; Gillis et al. 2009), individual-level heterosis (i.e., hybrid vigor; Facon et al. 2005), or both (Facon et al. 2005). Both phenomena can increase the invasiveness of non-native organisms, namely their ability to establish and spread in their novel range (Facon et al. 2005; Drake 2006; Crawford and Whitney 2010; Hahn and Rieseberg 2017; Wagner et al. 2017; Smyser et al. 2020).

The Panther Chameleon, Furcifer pardalis (Cuvier 1829), is native to Madagascar (Raxworthy et al. 2002). This species was first discovered in Florida in 2008 (Krysko et al. 2011), is now established (Krysko et al. 2016), and has been reported or documented by voucher specimens from seven counties: Broward (Rochford et al. 2013), Charlotte (MorphoBank M782844), DeSoto (Krysko et al. 2011), Miami-Dade (UF-Herpetology 178186), Orange (UF-Herpetology 191357), Polk (UF-Herpetology 190325), and Sarasota (this study).
Panther Chameleons exhibit striking sexual dimorphism, with adult male coloration varying from blue, green, or gray to brown or red, whereas adult females are generally drab tan or brown (Krysko et al. 2019). Moreover, the color polymorphism exhibited by adult males of allopatric Panther Chameleon populations in Madagascar is spatially structured and reflects underlying interpopulation genetic diversity (Grbic et al. 2015). Using mitochondrial DNA (mtDNA), Grbic et al. (2015) identified and mapped the distributions of three major native-range Panther Chameleon clades (lineages): Clade $A$, from northwestern Madagascar and the islands of Nosy Be and Nosy Komba; Clade B, present in northern Madagascar and along the northeastern coast; and Clade C, which is divisible into two subclades, C1 and C2, from the eastern coast and the island of Nosy Boraha (Isle Sainte Marie). Grbic et al. (2015) also used machine learning to create a color-based identification key, which can be used to assign adult male Panther Chameleons to a native clade/subclade. Although Panther Chameleons are known to change color seasonally (Grbic et al. 2015, Figure S5) or in
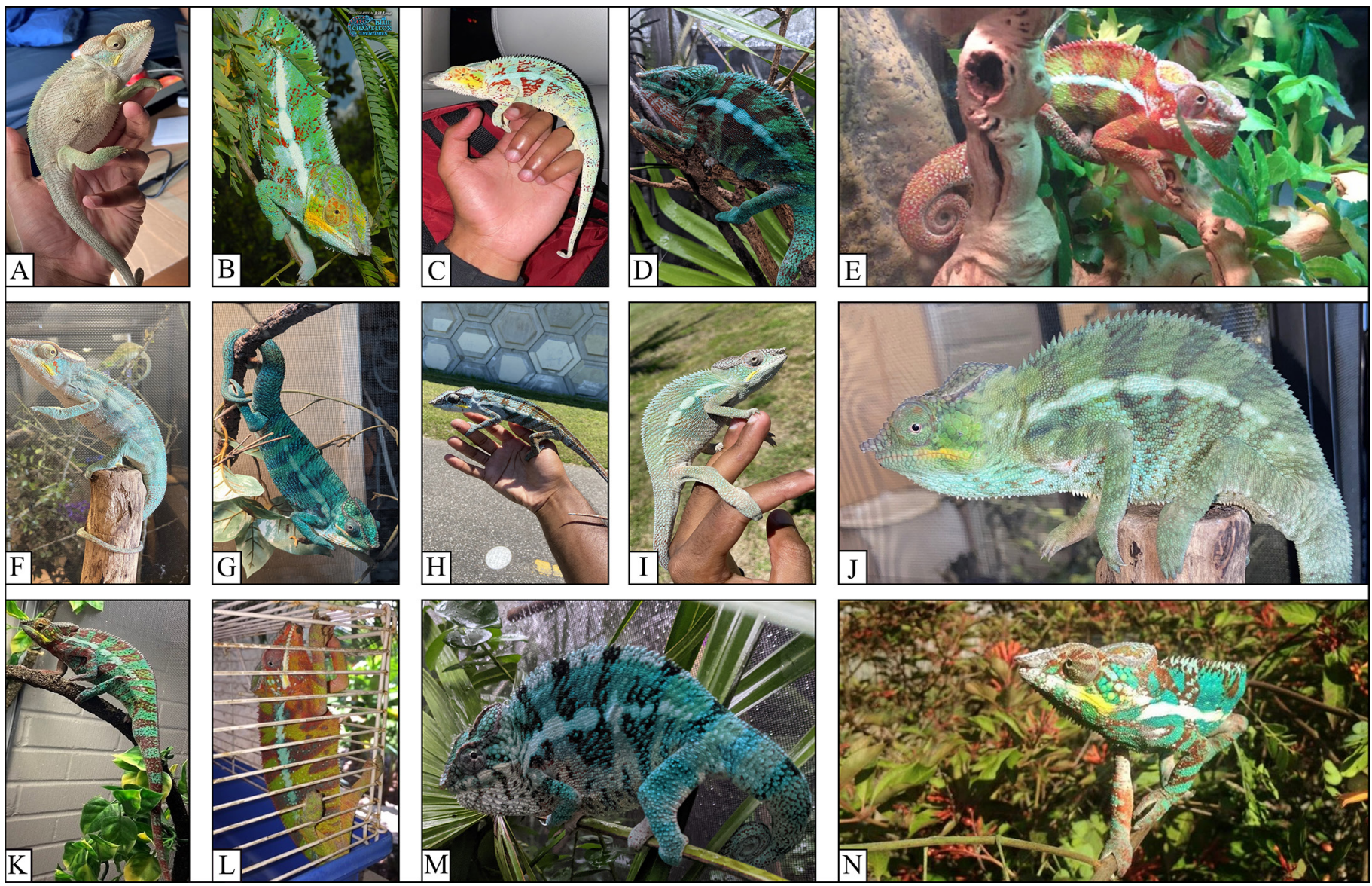

Fig. 2. Adult male Panther Chameleons (Furcifer pardalis) from Florida, USA, analyzed in this study: (A) Orange County, photograph by Brian T. Fridie; (B) south of Arcadia, DeSoto Couty, UF-Herpetology image voucher 153489; (C) Sarasota County, photograph by Brian T. Fridie; (D) Orange County, photograph by Natalie M. Claunch; (E) Coconut Creek, Broward County, UF-Herpetology image voucher 170514; (F) Orange County, photograph by Brian T. Fridie; (G) Orange County, photograph by Natalie M. Claunch; (H) Orange County, photograph by Brian T. Fridie; (I) Orange County, photograph by Brian T. Fridie; (J) Sarasota County, photograph by Brian T. Fridie; (K) Babson Park, Polk County, photograph by Brian T. Fridie; (L) Homestead, Miami-Dade County, UF-Herpetology image voucher 178186b; (M) Orange County, photograph by Natalie M. Claunch; (N) Babson Park, Polk County, EDDMaps Record ID 4766953. The complete set of images included in this study is archived in Morphobank (see text). 
response to stress (Teyssier et al. 2015), geographic differences in adult male base coloration nevertheless remain sufficiently consistent to serve as a useful tool for inferring native-range provenance (Ferguson et al. 2004; Grbic et al. 2015).

In this study, we used a modified version of the identification key from Grbic et al. (2015) (Fig. 1) to classify the likely clade and origin of 20 introduced adult male Panther Chameleons from Florida (Figs. 1-2). All photographs used for analysis are archived in MorphoBank (O'Leary and Kaufman 2011) under MorphoBank Project 4022 (accessible at http://morphobank.org/permalink/?P4022). Six of the seven counties from which the species has reliably been reported were included in our analysis (Table 1), the exception being Charlotte County, for which no suitable photographs were available. We have withheld exact locations in order to protect private landowners, as members of the public frequently trespass on private property in Florida in order to collect chameleons (Edwards et al. 2014).

We unambiguously assigned multiple chameleons to both Clade A ( $\mathrm{n}=5 ; 25 \%)$ and Clade C/Subclade C1 ( $\mathrm{n}=2$; $10 \%$ ), supporting the introduction of multiple native-range lineages of Panther Chameleon to Florida. Nevertheless, the majority of chameleons $(\mathrm{n}=13 ; 65 \%)$ were classified as belonging to either Clade $\mathrm{B}$ or Clade $\mathrm{C} / \mathrm{Subclade} \mathrm{C} 2$ but could not be more accurately assigned (Table 1; Fig. 3). Ferguson et al. (2004) found no differences in coloration between adult male Panther Chameleons from Antsiranana (Diego Suarez) in the extreme north of Madagascar and Sambava on the northeastern coast (Fig. 3). Interestingly, Antsiranana Panther Chameleons belong to Clade B, whereas Sambava Panther Chameleons belong to Clade C/Subclade $\mathrm{C} 2$, with the transition zone apparently occurring just north of Sambava (Fig. 3; Grbic et al. 2015). The concordance between the findings of Ferguson et al. (2004) and Grbic et al. (2015) implies that ambiguous assignment probably stems from a genuine lack of differences in color between adult male Panther Chameleons from Clade B and Clade C/Subclade C2, rather than a shortcoming of the Grbic et al. (2015) identification key. Clade B Panther Chameleons are distributed widely in northern and northeastern Madagascar (Fig. 3; Grbic et al. 2015), including areas known to serve as hubs for the international reptile trade (e.g., Antsiranana; Fieldsend et al. 2021). Consequently, suggesting that Clade B chameleons could be present in Florida is reasonable, as is the assumption that all three major native-range clades have been introduced to the state.

Probable sympatry between the major clades of Panther Chameleons was identified in Orange, Polk, and Sarasota Counties (Table 1; Fig. 3), highlighting the potential for clade hybridization in Florida. Hybrid Panther Chameleons also are likely to be present in Florida if individuals from distinct native-range lineages have mated in captivity (Grbic et al. 2015) and produced hybrid offspring that have subsequently escaped or been illegally released. Hybrids could conceivably manifest novel color patterns not recorded in the native range, making their classification difficult or impossible; thus, the large percentage of ambiguously assigned chameleons in this study could in part stem from the presence of hybrids.

Hybridization or admixture between multiple nativerange lineages can result in elevated population-level genetic diversity (Kolbe et al. 2007; Dlugosch and Parker 2008; Gillis et al. 2009), which can increase the invasiveness of non-native organisms (Drake 2006; Crawford and Whitney 2010; Hahn and Rieseberg 2017; Wagner et al. 2017; Smyser et al. 2020), for instance by relieving genetic bottlenecks and increasing adaptive potential (Lavergne and Molofsky 2007). In some cases, in situ hybridization between multiple non-native taxa actually leads to the emergence of distinct hybrid lineages that outcompete parental forms (Pysek et al. 2003; Suehs et al. 2004; Facon et al. 2005), a phenomenon for which indi-

Table 1. Summary of male Panther Chameleons (Furcifer pardalis) from Florida, USA, analyzed in this study ( $\mathrm{n}=20$ ). Clade names based on Grbic et al. (2015). Note that this table represents the number of adult male Panther Chameleons from each Florida county for which suitable photographic records were available, and does not necessarily represent the total number of records from a given county. All county records are from a single contiguous population, except Miami-Dade County, where the two chameleons were collected approximately 7 $\mathrm{km}$ apart.

\begin{tabular}{|c|c|c|c|c|}
\hline County & $\begin{array}{l}\text { Sample } \\
\text { Size }\end{array}$ & Clade A & $\begin{array}{c}\text { Clade B or } \\
\text { Clade C/Subclade C2 }\end{array}$ & $\begin{array}{c}\text { Clade C/ } \\
\text { Subclade C1 }\end{array}$ \\
\hline Broward & 2 & 0 & 2 & 0 \\
\hline DeSoto & 1 & 1 & 0 & 0 \\
\hline Miami-Dade & 2 & 1 & 1 & 0 \\
\hline Orange & 9 & 1 & 7 & 1 \\
\hline Polk & 3 & 1 & 2 & 0 \\
\hline Sarasota & 3 & 1 & 1 & 1 \\
\hline Total (\%) & $20(100 \%)$ & $5(25 \%)$ & $13(65 \%)$ & $2(10 \%)$ \\
\hline
\end{tabular}




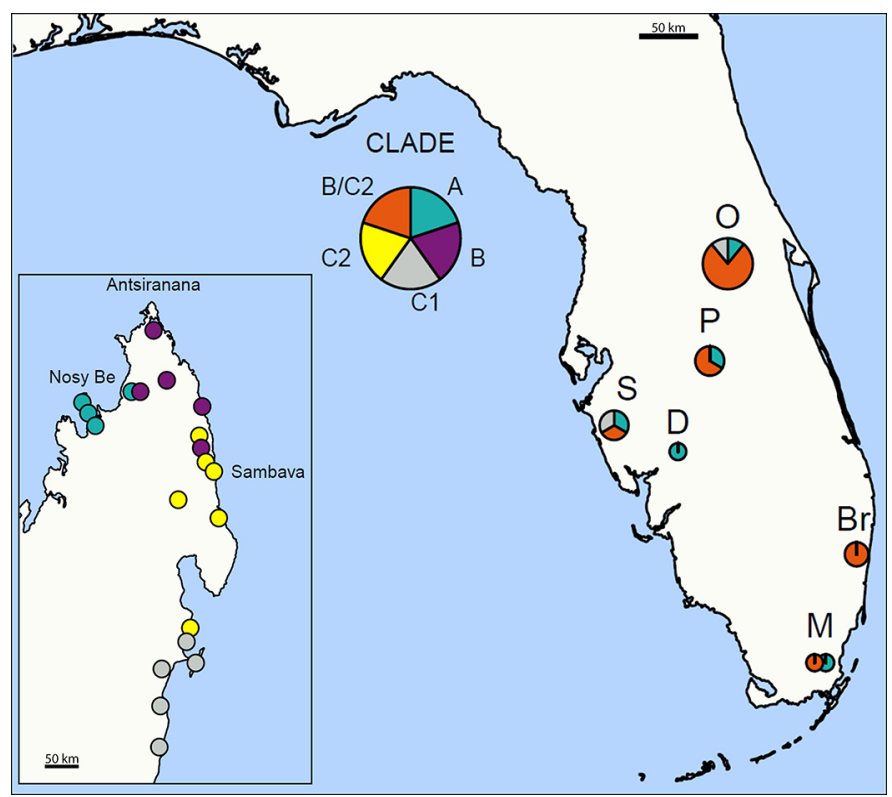

Fig. 3. Map of Florida, USA, showing the approximate locations of adult male Panther Chameleons (Furcifer pardalis) analyzed in this study. Colors correspond to the mitochondrial clades to which chameleons were assigned based on their adult coloration following Grbic et al. (2015) (see Fig. 1). Map circle sizes correspond to population sample sizes $(n=1-9)$, with circle segment coloration representing the proportion of chameleons assigned to each clade. Abbreviations above circles correspond to counties: $\mathrm{Br}=$ Broward; $\mathrm{D}=$ DeSoto; $\mathrm{M}=$ Miami-Dade; $\mathrm{O}=$ Orange; $\mathrm{P}=$ Polk; $S=$ Sarasota. Inset: map of northern Madagascar showing the approximate native-range distributions of the clades, generated using data from the Supplementary Materials of Grbic et al. (2015). Circle sizes on the Madagascar map do not correspond to sample sizes.

vidual-level heterosis can be the predominant driving force (e.g., Facon et al. 2005). Conversely, hybridization can hinder invasive success if it leads to hybrid inferiority (Macagno et al. 2021) or hybrid sterility (Turner et al. 2014). Ferguson et al. (2004) noted that some hybrid Panther Chameleons show reduced fitness, and detailed the case of a hybrid male Panther Chameleon with one parent from Nosy Be (inferred Clade A; Grbic et al. 2015) and one parent from the East Coast (inferred Clade C; Grbic et al. 2015) that was apparently completely sterile (Ferguson et al. 2004, Plate 4.63).

In conclusion, our study supports the introduction of multiple native-range Panther Chameleon lineages to Florida, and provides evidence that hybrids may already be present in the state. Given the aforementioned potential for hybridization and admixture to affect invasiveness, we suggest that further study is warranted to confirm the presence of hybrid individuals or lineages, and to characterize the fitness of any hybrids relative to parental forms.

\section{Acknowledgement}

We thank Coleman M. Sheehy III (Division of Herpetology, Florida Museum of Natural History, University of Florida) for providing Furcifer pardalis image vouchers.

\section{Literature Cited}

Crawford, K. and K. Whitney. 2010. Population genetic diversity influences colonization success. Molecular Ecology 19: 1253-1263. https://doi.org/10.1111/ j.1365-294X.2010.04550.x.

Dlugosch, K.M. and I.M. Parker. 2008. Founding events in species invasions: Genetic variation, adaptive evolution, and the role of multiple introductions. Molecular Ecology 17: 431-449. https://doi.org/10.1111/j.1365294X.2007.03538.x.

Drake, J.M. 2006. Heterosis, the catapult effect and establishment success of a colonizing bird. Biology Letters 2: 304-307. https://doi.org/10.1098/ rsbi.2006.0459.

Edwards, J.R., M.R. Rochford, F.J. Mazzotti, and K.L. Krysko. 2014. New county record for the Veiled Chameleon (Chamaeleo calyptratus Duméril and Bibron 1851), in Broward County, Florida, with notes on intentional introductions of chameleons in southern Florida. Reptiles \& Amphibians 21: 83-85.

Facon, B., P. Jarne, J.P. Pointier, and P. David. 2005. Hybridization and invasiveness in the freshwater snail Melanoides tuberculata: hybrid vigour is more important than increase in genetic variance. Journal of Evolutionary Biology 18: 524-535. https://doi.org/10.1111/j.1420-9101.2005.00887.x.

Ferguson, G.W., J.B. Murphy, J-B. Ramanamanjato, and A.P. Raselimanana. 2004. The Panther Chameleon: Color Variation, Natural History, Conservation, and Captive Management. Krieger Publishing Company, Malabar, Florida, USA.

Fieldsend, T.W., K.L. Krysko, P. Sharp, and T.M. Collins. 2021. Provenance and genetic diversity of the non-native geckos Phelsuma grandis Gray 1870 and Gekko gecko (Linnaeus 1758) in southern Florida, USA. Biological Invasions 23: 1649-1662. https://doi.org/10.1007/s10530-021-02463-1.

Gillis, N.K., L.J. Walters, F.C. Fernandes, and E.A. Hoffman. 2009. Higher genetic diversity in introduced than in native populations of the mussel Mytella charruana: evidence of population admixture at introduction sites. Diversity and Distributions 15: 784-795. https://doi.org/10.1111/j.14724642.2009.00591.x.

Grbic, D., S.V. Saenko, T.M. Randriamoria, A. Debry, A.P. Raselimanana, and M.C. Milinkovitch. 2015. Phylogeography and support vector machine classification of color variation in panther chameleons. Molecular Ecology 24: 3455-3466. https://doi.org/10.1111/mec.13241.

Hahn, M.A. and L.H. Rieseberg. 2017. Genetic admixture and heterosis may enhance the invasiveness of common ragweed. Evolutionary Applications 10: 241-250. https://doi.org/10.1111/eva.12445.

Kolbe, J.J., R.E. Glor, L.R. Schettino, A.C. Lara, A. Larson, and J.B. Losos. 2007. Multiple sources, admixture, and genetic variation in introduced Anolis lizard populations. Conservation Biology 21: 1612-25. https://doi.org/10.1111/ j.1523-1739.2007.00826.x.

Krysko, K.L., J.P. Burgess, M.R. Rochford, C.R. Gillette, D. Cueva, K. Enge, L.A. Somma, J.L. Stabile, D.C. Smith, J.A. Wasilewski, G.N. Kieckheffer III, M.C. Granatosky, and S.V. Nielsen. 2011. Verified non-indigenous amphibians and reptiles in Florida from 1863 through 2010: Outlining the invasion process and identifying invasion pathways and stages. Zootaxa 3028: 1-64. https://doi.org/10.11646/zootaxa.3028.1.1.

Krysko, K.L., L.A. Somma, D.C. Smith, C.R. Gillette, D. Cueva, J.A. Wasilewski, K.M. Enge, S.A. Johnson, T.S. Campbell, J.R. Edwards, M.R. Rochford, R. Tompkins, J.L. Fobb, S. Mullin, C.J. Lechowicz, D. Hazelton, and A. Warren. 2016. New verified nonindigenous amphibians and reptiles in Florida through 2015, with a summary of over 152 years of introductions. Reptiles \& Amphibians 23: 110-143.

Krysko, K.L., K.M. Enge, and P.E. Moler. 2019. Amphibians and Reptiles of Florida. University of Florida Press, Gainesville, Florida, USA.

Lavergne, S. and J. Molofsky. 2007. Increased genetic variation and evolutionary potential drive the success of an invasive grass. Proceedings of the National Academy of Sciences 104: 3883-3888. https://doi.org/10.1073/ pnas.0607324104.

Macagno, A.L., T.J. Edgerton, and A.P. Moczek. 2021. Incipient hybrid inferiority between recently introduced, diverging dung beetle populations. Biological Journal of the Linnean Society 132: 931-944. https://doi.org/10.1093/biolinnean/blaa228.

O'Leary, M.A. and S. Kaufman. 2011. MorphoBank: phylophenomics in the "cloud." Cladistics 27: 529-537. https://doi.org/10.1111/j.10960031.2011.00355.x.

Pysek, P., J.H. Brock, K. Bimova, B. Mandak, V. Jarosik, I. Koukolikova, J. Pergl, and J. Stepanek. 2003. Vegetative regeneration in invasive Reynoutria (Polygonaceae) taxa: the determinant of invasibility at the genotype level. 
American Journal of Botany 90: 1487-1495. https://doi.org/10.3732/ ajb.90.10.1487.

Raxworthy, C.J., M.R.J. Forstner, and R.A. Nussbaum. 2002. Chameleon radiation by oceanic dispersal. Nature 415: 784-787. https://doi.org/10.1038.415784a.

Rochford, M.R., J.R. Edwards, P.L. Howell, J.K. Eckles, L.A. Barraco, L.L. Connor, M.J. Curtis, K.L. Krysko, and F.J. Mazzoti. 2013. The Panther Chameleon, Furcifer pardalis (Cuvier 1829) (Chamaeleonidae), another introduced chameleon species in Florida. Reptiles \& Amphibians 20: 205-207.

Smyser, T.J., M.A. Tabak, S.B. Robeson, and H. Blackburn. 2020. Mixed ancestry from wild and domestic lineages contributes to the rapid expansion of invasive feral swine. Molecular Ecology 29: 1103-1119. https://doi.org/10.1111/ mec. 15392.

Suehs, C.M., L. Affre, and F. Medail. 2004. Invasion dynamics of two alien
Carpobrotus (Aizoaceae) taxa on a Mediterranean island: I. Genetic diversity and introgression. Heredity 92: 31-40. https://doi.org/10.1038/ sj.hdy. 6800374

Teyssier, J., S.V. Saenko, D. van der Marel, and M.C. Milinkovitch. 2015. Photonic crystals cause active color change in chameleons. Nature Communications 6 : 1-7. https://doi.org/10.1038/ncomms7368.

Turner, L.M., M.A. White, D. Tautz, and B.A. Payseur. 2014. Genomic networks of hybrid sterility. PLoS Genetics 10: e1004162. https://doi.org/10.1371/journal.pgen.1004162.

Wagner, N.K., B.M. Ochocki, K.M. Crawford, A. Compagnoni, and T.E. Miller. 2017. Genetic mixture of multiple source populations accelerates invasive range expansion. Journal of Animal Ecology 86: 21-34. https://doi. org/10.1111/1365-2656.12567. 